\title{
On the theory and phenomenology of spacetime symmetries at the Planck scale
}

\author{
Giovanni Amelino-Camelia* \\ Dipart. Fisica Univ. La Sapienza and Sez. Romal INFN \\ P.le Moro 2, I-00185 Roma, Italy E-mail: amelino@roma1. infn. it
}

\begin{abstract}
I discuss some alternative scenarios for the fate of Poincaré/Lorentz symmetry at the Planck scale. In particular, I provide a phenomenologist characterization, rather different from math-oriented alternatives of recent fashion, of the doubly-special-relativity idea. I also discuss a few examples of of experiments that can be valuable for these studies, in some cases also able to discriminate between scenarios with a deformation of symmetries, of the specific type advocated in doublyspecial relativity, and ordinary symmetry-breaking scenarios.
\end{abstract}

From Quantum to Emergent Gravity: Theory and Phenomenology

June 11-15 2007

Trieste, Italy

\footnotetext{
* Speaker.
} 


\section{Different perspectives on the fate of Poincaré symmetry at the Planck scale}

While the quantum-gravity problem clearly motivates a more general investigation of spacetime symmetries, most related work so far has focused on the fate of Poincaré symmetries in the "Minkowski limit" (as defined in Ref. [1]) of quantum gravity.

While of course a key exercise is to analyze proposed solutions of the quantum/gravity problem, focusing on Poincaré-symmetry/Minkowski-limit issues, it is important to realize that motivation for placing Poincaré symmetry under scrutiny is found in the structure of the quantum-gravity problem, even before starting to consider possible solutions. Surprisingly, the description of how this motivation emerges depends strongly on the scientific background of the author who is elaborating this intuition, but most authors would agree on the end result.

It is probably fair to state that each quantum-gravity research line can be connected with one of three perspectives on the problem: the particle-physics perspective, the General-Relativity perspective and the condensed-matter perspective. And each of these perspectives provides its on motivation for investigating Poincaré symmetry at the Planck scale.

From a particle-physics perspective it is natural to attempt to reproduce as much as possible the successes of the Standard Model of particle physics. One is tempted to see gravity simply as one more gauge interaction. From this particle-physics perspective a natural solution of the quantumgravity problem should have its core features described in terms of graviton-like exchange in a background classical spacetime. Indeed this structure is found in String Theory, the most developed among the quantum-gravity approaches that originate from a particle-physics perspective. From this perspective there is no a priori reason to contemplate departures from Poincaré symmetry: if classical Minkowski spacetime is an acceptable background then one would also not expect surprises for what concerns Poincare symmetry. Still, it is noteworthy that, even assuming that it makes sense to consider a classical background spacetime, the fate of Poincare symmetry in String Theory is somewhat uncertain: it has been found (see, e.g., Ref. [2] and references therein) that under appropriate conditions (a vacuum expectation value for certain tensor fields) Poincaré symmetry is broken.

The General-Relativity perspective naturally leads us to reject the use of a background spacetime. According to General Relativity the evolution of particles and the structure of spacetime are selfconsistently connected: rather than specify a spacetime arena (a spacetime background) beforehand, the dynamical equations determine at once both the spacetime structure and the evolution of particles. Although less publicized, there is also growing awareness of the fact that, in addition to the concept of background independence, the development of General Relativity relied heavily on the careful consideration of the in-principle limitations that measurement procedures can encounter. In light of the various arguments suggesting that, whenever both quantum mechanics and General Relativity are taken into account, there should be an in-principle Planck-scale limitation to the localization of a spacetime point (an event), the general-relativity perspective invites one to renounce to any direct reference to a classical spacetime $[3,4,5,6,7]$. Indeed this requirement that spacetime be described as fundamentally nonclassical ("fundamentally quantum"), the requirement that the in-principle measurability limitations be reflected by the adoption of a corresponding measurability-limited description of spacetime, is another element of intuition which is guiding quantum-gravity research from the general-relativity perspective. This naturally leads one 
to consider nonclassical (e.g. discretized [8, 9] or noncommutative [2, 3, 10, 11, 12]) spacetimes, in which case of course one must of course reconsider the status of Poincaré symmetry.

The third possibility is a condensed-matter perspective on the quantum-gravity problem (see, e.g., Refs. $[13,14]$ ), in which spacetime itself is seen as a sort of emerging critical-point entity. Condensed-matter theorists are used to describe the degrees of freedom that are measured in the laboratory as collective excitations within a theoretical framework whose primary description is given in terms of much different, and often practically inaccessible, fundamental degrees of freedom. Close to a critical point some symmetries arise for the collective-excitations theory, which do not carry the significance of fundamental symmetries, and are in fact lost as soon as the theory is probed somewhat away from the critical point. Notably, some familiar systems are known to exhibit special-relativistic invariance in certain limits, even though, at a more fundamental level, they are described in terms of a nonrelativistic theory. Clearly for the condensed-matter perspective on the quantum-gravity problem it is natural to see the familiar classical continuous Poincaré symmetry only as an approximate symmetry.

So, while of course it is also not unlikely that Poincaré symmetry might survive in quantumgravity's Minkowski limit, the motivation for placing under scrutiny Poincaré symmetry at the Planck scale is rather strong. Unfortunately, it is extremely hard to gain experimental access, even indirectly, to the Planck-scale regime (as characterized, e.g., by the tiny Planck length $L_{p} \sim$ $10^{-35} \mathrm{~m}$ ). As the content of the following sections will suggest, at least over a reasonable time of,say, a few decades, even adopting a very optimistic viewpoint, we cannot imagine having more than 3 or 4 relevant data analyses, on which to ground our deductions for the fate of Poincaré symmetry. In this respect it is important to establish that the relevant phenomenology has the ability to discriminate between different (but similar) scenarios. And it is for this reason that here I stress the difference between the doubly-special-relativity scenario and ordinary symmetrybreaking scenarios.

The next section is devoted to doubly-special relativity [15], which I describe (as originally proposed) as a scenario for Planck-scale physics, rather than one or another choice of formalism. And I show that the doubly-special-relativity idea can be falsified, a rare example of falsifiable proposal for Planck-scale physics.

Section 3 may be used as a compact point of entry in the literature on the phenomenology of Planck-scale departures from Poincaré (Lorentz) symmetry. I do not give detailed accounts (which are provided in several detailed reviews, such as the ones in Refs. $[16,17,18,19])$ but I try to touch on a representative subset of the ideas the community is pursuing, and in doing so I try to show as clearly as possible how important it is to rely on some carefully tailored, commonly adopted, test theories in mapping the progress of this phenomenology. And I stress that in some cases the data can indeed discriminate between a doubly-special-relativity scenario and a symmetry-breaking scenario.

In Section 4 I mention certain issues that must be taken into account when performing some of these data analyses.

Some closing remarks are offered in Section 5. 


\section{Doubly-Special Relativity}

In preparation for the next Section, which focuses on the phenomenology of Planck-scale departures from Poincaré symmetry, I find it useful to provide here a short but self-contained introduction to "doubly-special relativity" (DSR). This is a scenario which I proposed only a few years ago[15], but is already rather extensively analyzed as an alternative to the standard scenario of Planck-scale effects that break Lorentz(/Poincaré) symmetry. Aa a result of this interest, at this point there are in the literature numerous attempts of review of DSR research, so one might think this section could be unnecessary. However, DSR is becoming different things to different authors, and the differences are rather significant from the "phenomenologist perspective" which I am here adopting. The DSR proposal, which originally provided a physics scenario for the Planck scale, is now often identified with a certain (rather vaguely defined) mathematical framework, whether or not this mathematical framework turns out to be compatible with the DSR principles.

This recent mathematical twist of the DSR literature may well some day mature into a powerful tool for quantum gravity research, perhaps both at the conceptual level and for what concerns phenomenology, but at present it is certainly of no use for phenomenology (and even the conceptual side is only at an early stage of development). Instead for the objectives I have for these notes my original DSR proposal is rather valuable since it provides a rare example of a Planck-scale physics idea that is powerful enough to make definite falsifiable predictions (even without any knowledge of the correct formalism that should implement it!). This is stressed in particular in the part of the next section devoted to photon stability.

\subsection{Motivation}

I introduced the doubly-special-relativity scenario as a sort of alternative perspective on the results on Planck-scale departures from Lorentz symmetry which had been reported in numerous articles [20,21, 22, 23, 24, 25, 26] between 1997 and 2000. These studies were advocating a Planck-scale modification of the energy-momentum dispersion relation, usually of the form $E^{2}=p^{2}+m^{2}+\eta L_{p}^{n} p^{2} E^{n}+O\left(L_{p}^{n+1} E^{n+3}\right)$, on the basis of preliminary findings in the analysis of several formalisms in use for Planck-scale physics. The complexity of the formalisms is such that very little else was known about their physical consequences, but the evidence of a modification of the dispersion relation was becoming robust. In all of the relevant papers it was assumed that such modifications of the dispersion relation would amount to a breakup of Lorentz symmetry, with associated emergence of a preferred class of inertial observers (usually identified with the natural observer of the cosmic microwave background radiation). I was intrigued by a striking analogy between these developments and the developments which led to the emergence of Special Relativity, now more than a century ago. In Galilei Relativity there is no observer-independent scale, and in fact the energy-momentum relation is written as $E=p^{2} /(2 m)$. As experimental evidence in favour of Maxwell equations started to grow, the fact that those equations involve a fundamental velocity scale appeared to require the introduction of a preferred class of inertial observers. But in the end we figured out that the situation was not demanding the introduction of a preferred frame, but rather a modification of the laws of transormation between inertial observers. Einstein's Special Relativity introduced the first observer-independent relativistic scale (the velocity scale $c$ ), its dispersion relation takes the form $E^{2}=c^{2} p^{2}+c^{4} m^{2}$ (in which $c$ plays a crucial role for what con- 
cerns dimensional analysis), and the presence of $c$ in Maxwell's equations is now understood as a manifestation of the necessity to deform the Galilei transformations.

I argued in Refs. [15] that it is not implausible that we might be presently confronted with an analogous scenario. Research in quantum gravity is increasingly providing reasons of interest in Planck-scale modifications of the dispersion relation, of the type mentioned above, and, while it was customary to assume that this would amount to the introduction of a preferred class of inertial frames (a "quantum-gravity ether"), the proper description of these new structures might require yet again a modification of the laws of transformation between inertial observers. The new transformation laws would have to be characterized by two scales $\left(c\right.$ and $\left.L_{p}\right)$ rather than the single one (c) of ordinary Special Relativity.

\subsection{Defining the DSR scenario}

The "historical motivation" described above leads to a scenario for Planck-scale physics which is not intrinsically equipped with a mathematical formalism for its implementation, but still is rather well defined. With Doubly-Special Relativity one looks for a transition in the Relativity postulates, which should be largely analogous to the Galilei $\rightarrow$ Einstein transition. Just like it turned out to be necessary, in order to describe high-velocity particles, to set aside Galilei Relativity (with its lack of any characteristic invariant scale) and replace it with Special Relativity (characterized by the invariant velocity scale $c$ ), it is at least plausible that, in order to describe ultra-high-energy particles, we might have to set aside Special Relativity and replace it with a new relativity theory, a DSR, with two characteristic invariant scales, a new small-length/large-momentum scale in addition to the familiar velocity scale.

A theory will be compatible with the DSR principles if there is complete equivalence of inertial observers (Relativity Principle) and the laws of transformation between inertial observers are characterized by two scales, a high-velocity scale and a high-energy/short-length scale. Since in DSR one is proposing to modify the high-energy sector, it is safe to assume that the present operative characterization of the velocity scale $c$ would be preserved: $c$ is and should remain the speed of massless low-energy particles ${ }^{1}$. Only experimental data could guide us toward the operative description of the second invariant scale $\lambda$, although its size is naturally guessed to be somewhere in the neighborhood of the Planck length $L_{p}$.

As a result of the "historical context" described in the preceeding subsection most authors have explored the possibility that the second relativistic invariant be introduced through a modifications of the dispersion relation. This is a reasonable choice but it would be incorrect at present to identify (as often done in the literature) the DSR proposal with the proposal of observer-independent modifications of the dispersion relation. For example the dispersion relation might not be modified but there might instead be an observer-independent bound on the accuracy achievable in the measurement of distances.

In the search of a first example of formalism compatible with the DSR principles much work has been devoted to the study of $\kappa$-Minkowski. There are good reasons for this [1, 15], but once

\footnotetext{
${ }^{1}$ Note however the change of perspective imposed by the DSR idea: within Special Relativity $c$ is the speed of all massless particles, but Special Relativity must be perceived as a low-energy theory (as viewed from the DSR perspective) and in taking Special Relativity as starting point for a high-energy deformation one is only bound to preserving $c$ as the speed of massless low-energy particles
} 
again it would be incorrect to identify the DSR idea with $\kappa$-Minkowski. Of course we may one day stumble upon a very different formalism which is compatible with the DSR principles. And even within research on $\kappa$-Minkowski it must be noticed that the same mathematics can be used to obtain pictures which very clearly violate the DSR principles. For example some authors introduce theories in $\kappa$-Minkowski in a way that leads to a law of conservation of energy-momentum based on a naive substitution of the usual sum rule with the "coproduct" sum rule, but this amounts $[1,15]$ to breaking (rather than deforming) the Poincaré symmetries.

\section{Some aspects of the phenomenology of Planck-scale departures from Poincaré symmetry}

In this section I comment on some aspects of recent phenomenology work on departures from Poincaré symmetry, mostly as codified in modifications of the energy-momentum dispersion relation. I will start by stressing that the same modified dispersion relation can be introduced in very different test theories, leading to completely different physical predictions. But I also argue that for most of the ways in which a modified dispersion relation could manifest itself we do have at least some hope of experimental study.

\subsection{On the test theories with modified dispersion relation}

The majority (see, e.g., Refs. [20, 21, 22, 23, 24, 25, 26]) of studies concerning Planck-scale modifications of the dispersion relation adopt the phenomenological formula

$$
m^{2} \simeq E^{2}-\vec{p}^{2}+\eta \vec{p}^{2}\left(\frac{E^{n}}{E_{p}^{n}}\right)+O\left(\frac{E^{n+3}}{E_{Q G}^{n+1}}\right),
$$

with real $\eta$ (assumed to be of order $|\eta| \sim 1$ ) and integer $n$.

There is a this point a very large literature on the associated phenomenology, but I want to stress that actually some of the different phenomenological studies that compose this literature introduce this type of dispersion relation within different test theories. The limits obtained within different test theories are of course not to be compared. The same parametrization of the dispersion relation, if introduced within different test theories, actually gives rise to independent sets of parameters.

The potential richness of this phenomenology, for what concerns the development of test theories, mainly originates from the need to specify, in addition to the form of the dispersion relation, several other structural properties of the test theory.

It is necessary to state whether the theory is still "Hamiltonian", in the sense that the velocity along the $x$ axis is obtained from the commutator with an Hamiltonian $(v \sim[x, H])$ and whether the Heisenberg commutator preserves its standard form $([x, p] \sim \hbar)$. Especially this second concern is rather significant since some of the heuristic arguments which are used to motivate the presence of modified dispersion relation at the Planck scale also suggest that the Heisenberg commutator should be correspondingly modified.

Then the test theory should formulate a law of energy-momentum conservation. For example, some types of spacetime noncommutativity which contributed to interest in modified dispersion 
relations appear to be such to require also an accompanying modification of the law of energymomentum conservation. And in particular a link between modification of the dispersion relation and associated modification of the law of energy-momentum conservation is required by the DSR principles (see below).

And one should keep clearly separate the test theories that intend to describe only kynematics and the ones that also adopt a scheme for Planck-scale dynamics. For example, in Loop Quantum Gravity and some noncommutative spacetimes which provided motivation for considering modifications of the dispersion relation, while we might be close to have a correct picture of kinematics it appears that we are still far from understanding Planck-scale corrections to dynamics ${ }^{2}$

Elsewhere $[19,27]$ I have tried to propose a handful of test theories that could provide a first level of language to handle this complexity. Here I shall simply show how in different phenomenological studies based on modified dispersion relations one ends up making assumptions about the points listed above.

\subsection{Photon stability}

It has been recently realized (see, e.g., Refs. [28, 29, 30]) that when Lorentz symmetry is broken at the Planck scale there can be significant implications for certain decay processes. At the qualitative level the most significant novelty would be the possibility for massless particles to decay. Let us consider for example a photon decay into an electron-positron pair: $\gamma \rightarrow e^{+} e^{-}$. And let us analyze this process using the dispersion relation (3.1), for $n=1$, with unmodified law of energy-momentum conservation. One easily finds a relation between the energy $E_{\gamma}$ of the incoming photon, the opening angle $\theta$ between the outgoing electron-positron pair, and the energy $E_{+}$of the outgoing positron, which, for the region of phase space with $m_{e} \ll E_{\gamma} \ll E_{p}$, takes the form $\cos (\theta)=(A+B) / A$, with $A=E_{+}\left(E_{\gamma}-E_{+}\right)$and $B=m_{e}^{2}-\eta E_{\gamma} E_{+}\left(E_{\gamma}-E_{+}\right) / E_{p}\left(m_{e}\right.$ denotes of course the electron mass). The fact that for $\eta=0$ this would require $\cos (\theta)>1$ reflects the fact that if Lorentz symmetry is preserved the process $\gamma \rightarrow e^{+} e^{-}$is kinematically forbidden. For $\eta<0$ the process is still always forbidden, but for positive $\eta$ and $E_{\gamma} \gg\left(m_{e}^{2} E_{p} /|\eta|\right)^{1 / 3}$ one finds that $\cos (\theta)<1$ in certain corresponding region of phase space.

The energy scale $\left(m_{e}^{2} E_{p}\right)^{1 / 3} \sim 10^{13} \mathrm{eV}$ is not too high for astrophysics. The fact that certain observations in astrophysics allow us to establish that photons of energies up to $\sim 10^{14} \mathrm{eV}$ are not unstable (at least not noticeably unstable) could be used $[28,30]$ to set valuable limits on $\eta$ if $[19,27]$ the dispersion-relation modification is implemented within a framework that predicts (through some formulation of dynamics) a correspondingly large decay rate.

Notice however that by changing the form of the law of energy-momentum conservation, for fixed form of the dispersion relation, one can obtain very different results. This is best illustrated contemplating the possibility that such a dispersion relation be introduced within a DSR framework. First of all let us notice that any theory compatible with the DSR principle will have stable massless particles, so that by looking for massless-particle decay one could falsify the DSR idea. A threshold-energy requirement for massless-particle decay (such as the $E_{\gamma} \gg\left(m_{e}^{2} E_{p} /|\eta|\right)^{1 / 3}$ men-

\footnotetext{
${ }^{2}$ On the Loop Quantum Gravity side this is linked to the "classical limit problem", while for the relevant noncommutative spacetime the concern originates from failures to produce consistent theories of quantum matter fields in those spacetimes.
} 
tioned above) cannot of course be introduce as an observer-independent law, and is therefore incompatible with the DSR principles.

An analysis of the stability of massless particles that is compatible with the DSR principles can be obtained by combining the modification of the dispersion relation with an associated modification of the laws of energy-momentum conservation. The form of the new law of energy-momentum conservation can be derived from the requirement of being compatible both with the DSR principles and with the modification of the dispersion relation [15], and in particular for the $a \rightarrow b+c$ case which I am considering one arrives at $E_{\gamma} \simeq E_{+}+E_{-}-\lambda \vec{p}_{+} \cdot \vec{p}_{-}, \vec{p}_{\gamma} \simeq \vec{p}_{+}+\vec{p}_{-}-\lambda E_{+} \vec{p}_{-}-\lambda E_{-} \vec{p}_{+}$. Using these in place of ordinary conservation of energy-momentum one ends up with a result for $\cos (\theta)$ which is still of the form $(A+B) / A$ but now with $A=2 E_{+}\left(E_{\gamma}-E_{+}\right)+\lambda E_{\gamma} E_{+}\left(E_{\gamma}-E_{+}\right)$and $B=2 m_{e}^{2}$. Evidently this formula always gives $\cos (\theta)>1$, consistently with the fact that $\gamma \rightarrow e^{+} e^{-}$ is forbidden in DSR.

\subsection{Threshold anomalies}

Another opportunity to investigate Planck-scale departures from Lorentz symmetry is provided by certain types of energy thresholds for particle-production processes that are relevant in astrophysics. In particular, adopting a modified dispersion relation within a framework where the laws of energy-momentum conservation are unchanged one easily finds [21, 22, 23, 24] significant implications for the cosmic-ray spectrum. In fact, the "GZK cutoff", a key expected feature of the cosmic-ray spectrum, is essentially given by the threshold energy for cosmic-ray protons to produce pions in collisions with CMBR photons. In the evaluation of the threshold energy for $p+\gamma_{C M B R} \rightarrow p+\pi$ a correction term of order, say, $\eta \vec{p}^{2} E^{2} / E_{p}^{2}$ in the dispersion relation can be very significant. Whereas the classical-spacetime prediction for the GZK cutoff is around $5 \cdot 10^{19} \mathrm{eV}$, at those energies the Planck-scale correction to the threshold turns out [21, 22, 23, 24] to be of the order of $\eta E^{4} /\left(\varepsilon E_{p}^{2}\right)$, where $\varepsilon$ is the typical CMBR-photon energy. For positive values of $\eta$, even somewhat smaller than 1 , this amounts to an observably large shift of the threshold energy.

I should here stress that for the purpose of the point I am trying to convey in this section the study of threshold anomalies is not different from the study of the stability of massless particles: once again in the case in which the modified dispersion relation is combined with unmodified law of energy-momentum conservation one finds a striking effect. But the size of this effect can change significantly if one also allows a modification of the law of energy-momentum conservation.

\subsection{Time-of-travel analyses}

A wavelength dependence of the speed of photons is obtained from a modified dispersion relation, if one assumes the velocity to be still described by $v=d E / d p$. For the dispersion relation here considered one finds that at "intermediate energies" ( $m<E \ll E_{p}$ ) the velocity law will take the form

$$
v \simeq 1-\frac{m^{2}}{2 E^{2}}+\eta \frac{n+1}{2} \frac{E^{n}}{E_{p}^{n}} .
$$

On the basis of this formula one would find that two simultaneously-emitted photons should reach the detector at different times if they carry different energy. And this time-of-arrival-difference effect can be significant[20] in the analysis of short-duration gamma-ray bursts that reach us from cosmological distances. For a gamma-ray burst it is not uncommon that the time travelled before 
reaching our Earth detectors be of order $T \sim 10^{17} \mathrm{~s}$. Microbursts within a burst can have very short duration, as short as $10^{-3} s$, and this means that the photons that compose such a microburst are all emitted at the same time, up to an uncertainty of $10^{-3} \mathrm{~s}$. Some of the photons in these bursts have energies that extend at least up to the $\mathrm{GeV}$ range, and for two photons with energy difference of order $\Delta E \sim 1 \mathrm{GeV}$ a $\Delta E / E_{p}$ speed difference over a time of travel of $10^{17} s$ would lead to a difference in times of arrival of order $\Delta t \sim T \Delta \frac{E}{E_{p}} \sim 10^{-2} s$ which is significant (the time-of-arrival differences would be larger than the time-of-emission differences within a microburst).

It is well established that the sensitivities achievable [31] with the next generation of gammaray telescopes, such as GLAST [31], could allow to test very significantly (3.2) in the case $n=1$, by possibly pushing the limit on $|\eta|$ far below 1 . And, while probably beyond the reach of photon astrophysics, for the case $n=2$ neutrino astronomy may lead to valuable insight [32,33].

Notice however that in some test theories it may be incorrect to combine the limits obtained in this way with the limits obtained from the threshold analyses discussed above. The threshold analyses require a test theory with certain forms of the law of energy-momentum conservation, whereas the law of energy-momentum conservation is not relevant for the time-of-travel studies. On the other hand a test theory using the time-of-travel analyses, in adopting $v=d E / d p$, should have a standard form of the Heisenberg commutator (so that $x \rightarrow \partial / \partial p$ ) and assign a standard role to the Hamiltonian (so that $v \sim[x, H]$ )).

\subsection{Synchrotron radiation}

As observed recently in Ref. [34] (and discussed more carefully in the recent Ref. [35]), in the mechanism that leads to the production of synchrotron radiation a key role is played by the special-relativistic velocity law $v=d E / d p \simeq 1-m^{2} /\left(2 E^{2}\right)$. And an interesting observation is obtained by considering the velocity law (3.2) for the case $n=1$. Assuming that all other aspects of the analysis of synchrotron radiation remain unmodified at the Planck scale, one is led [34] to the conclusion that, if $\eta<0$, the energy/wavelength dependence of the Planck-scale term in (3.2) can affect the value of the cutoff energy for synchrotron radiation. This originates from the fact that according to (3.2), for $n=1$ and $\eta<0$, an electron cannot have a speed that exceeds the value $v_{e}^{\max } \simeq 1-(3 / 2)\left(|\eta| m_{e} / E_{p}\right)^{2 / 3}$, whereas in special relativity $v_{e}$ can take values arbitrarily close to 1. This may be used to argue that for negative $\eta$ the cutoff energy for synchrotron radiation should be lower than it appears to be suggested by certain observations of the Crab nebula [34].

In making use of this striking observation it is however important to notice that synchrotron radiation is due to the acceleration of the relevant electrons and therefore dynamics plays an implicit role in the derivation of the result [27]. From a field-theory perspective the process of synchrotronradiation emission is described in terms of Compton scattering of the electrons with the virtual photons of the magnetic field, confirming the need to include a description of some aspects of dynamics and of energy-momentum conservation (for the vertices in the Compton-scattering analysis).

\section{The issue of not-so-subleading correction terms}

I now want to consider some issues that may be particularly relevant when comparing different entries in the growing list of "experimental limits on Planck-scale spacetime-symmetry scenarios", 
of which I discussed a few examples in the previous section. As already clear by looking at the few examples I discussed, these limits are being obtained using two very different strategies. One strategy relies on very precise low-energy data. At low energies $E$ the ratios $\left(E / E_{p}\right)^{n}$, which set the expected magnitude of the effects, are usually horrifyingly small e.g. $E / E_{p} \sim 10^{-22}$ for particles with energies of a few MeVs, but in some cases [20] (at least for small $n$ ) data are found to be precise enough to provide the needed sensitivity. The other possibility is to use data at the highest energies accessible to us (for the relevant effect), so that the Planck-scale penalization is less severe. Typically the quality of the data quickly decreases as the energy scales under study increase, but the trade is in some cases apparently convenient: the lower precision of the data is often more than compensated by the larger expected magnitude of the effected. Most notably some analyses $[23,24,34]$ have focused on instances in which the formulas that characterize the effect under observation are such that the conjectured Planck-scale correction terms would be (at the relevant energy scales) stronger than some Planck-scale-independent terms also present in those formulas. When this happens the Planck scale effects can even change qualitatively the behaviour of the observables under study, and this allows one to use data of relatively low precision to obtain very strong experimental limits.

In this section I want to briefly summarize a point that will be articulated in more detail elsewhere [36], concerning the necessity to adopt some prudence when considering limits obtained using situations where the leading-order Planck-scale corrections dominate over some zero-th order terms. If some mechanism is allowing the leading-order Planck-scale correction to dominate over some zero-th order terms it is likely that that same mechanism would also be such that some "formally subleading" Planck-scale corrections, neglected in the analysis, are significant.

A first relevant example is provided [36] by studies considering a deformation of the speedenergy relation of the type

$$
v \simeq 1-\frac{m^{2}}{2 E^{2}}+\Delta\left(E, m ; E_{p}\right) \simeq 1-\frac{m^{2}}{2 E^{2}}-\eta \frac{E}{E_{p}}+\ldots,
$$

for ultrarelativistic $(E \gg m)$ particles. The truncation of the "quantum-gravity effect" $\Delta\left(E, m ; E_{p}\right)$ to the "leading-order" $E / E_{p}$ correction is not usually discussed in detail: most authors find sufficient to argue that the terms that are neglected would be suppressed by at least two powers of the inverse of the Planck scale and would therefore be inevitably too small to matter.

Using this type of truncation may indeed be "dangerous" in cases where a particle of mass $m$ has energy $E$ such that the correction term $E / E_{p}$ actually dominates over the "zero-th order term" $m^{2} /\left(2 E^{2}\right)$. Urrutia and I articulate this concern from various perspectives in Ref. [36], but for my purposes here it suffices to exhibit an explicit (although not necessarily compelling) example of all-order formula for $\Delta\left(E, m ; E_{p}\right)$ that would conflict with the proposed truncation. If, say, one had $\Delta\left(E, m ; E_{p}\right)=\eta E / E_{p}\left(\tanh \left[E^{6} /\left(m^{4} E_{p}^{2}\right)\right]-1\right)$ then the exact speed-energy relationship would be

$$
v \simeq 1-\frac{m^{2}}{2 E^{2}}+\eta \frac{E}{E_{p}}\left(\tanh \left(\frac{E^{6}}{m^{4} E_{p}^{2}}\right)-1\right),
$$

which at low energies still gives

$$
v \simeq 1-\frac{m^{2}}{2 E^{2}}+\eta\left(-\frac{E}{E_{p}}+\frac{E^{7}}{m^{4} E_{p}^{3}}\right) \simeq 1-\frac{m^{2}}{2 E^{2}}-\eta \frac{E}{E_{p}},
$$


since for $m^{2} /\left(2 E^{2}\right) \gg E / E_{p}$ one also has $\tanh \left(E^{6} /\left(m^{4} E_{p}^{2}\right)\right)-1 \simeq-1$, but as soon as $E$ is even just slightly above the value $\left(E_{p} m^{2}\right)^{1 / 3}$ one finds $\left(\tanh \left[E^{6} /\left(m^{4} E_{p}^{2}\right)\right]-1\right) \simeq 0\left(\right.$ e.g. for $E=1.5\left(E_{p} m^{2}\right)^{1 / 3}$ one has $\left.\left(\tanh \left[E^{6} /\left(m^{4} E_{p}^{2}\right)\right]-1\right) \simeq-2.6 \cdot 10^{-10}\right)$.

An equally clear illustration of our concerns can be provided [36] considering the case of analyses of particle-production reactions, in which, as mentioned, some authors have adopted a Planck-scale-corrected ultrarelativistic energy-momentum (dispersion) relation of the type

$$
p \simeq E-m^{2} /(2 E)+\delta\left(m, E ; E_{p}\right) \simeq E-\frac{m^{2}}{2 E}-\zeta \frac{E^{2}}{E_{p}} .
$$

Also this truncation of the "quantum-gravity effect" $\delta\left(E, m ; E_{p}\right)$ to the "leading-order" $E^{2} / E_{p}$ correction is not usually discussed in detail: again most authors find sufficient to argue that the terms that are neglected would be suppressed by at least two powers of the inverse of the Planck scale and would therefore be inevitably too small to matter.

A modification of threshold requirements for certain particle-physics reactions follows immediately from (4.4) (assuming the law of energy-momentum conservation is not Planck-scale modified). This is for example significant [22, 23, 24] for the process of electron-positron pair production in photon-photon collisions when one of the two incoming photons is hard (say a few $\mathrm{TeV}$ ) and the other incoming photon is very soft (or order, say, $10^{-2} \mathrm{eV}$ ). The Planck-scale-corrected prediction of absorption of multi- $\mathrm{TeV}$ photons by this mechanism differs very significantly from the standard formula, and in particular for some distant Blazars, whereas usually one expects little or no detections on Earth of multi-Tev photons (they should all disappear into electron-positron pairs as they travel from the Blazar to our detectors), the Planck-scale-corrected analysis would predict that multi- $\mathrm{TeV}$ photons are not allowed to produce electron-positron pairs and there is therefore nothing to prevent them from reaching our detectors. Since indirect evidence of absorption of multi- $\mathrm{TeV}$ photons is found in Blazar data, one could use this analysis to place strong constraints on $\zeta$. However, let us for example contemplate this specific ${ }^{3}$ next-to-leading form of the dispersion relation:

$$
p \simeq E-\frac{m^{2}}{2 E}-\zeta \frac{E^{2}}{E_{p}}+i \alpha \frac{E^{4}}{E_{p}^{3}} .
$$

This amounts to an extra term which goes like $E_{p}^{-3}$, and therefore is of the type one usually expects to be able to ignore safely with respect to the leading-order $E_{p}^{-1}$ term, but it is pure imaginary, and therefore automatically generates absorption. As shown in Ref. [36], the "formally subleading" term in this case is actually strong enough to change completely the outcome of the analysis, with

\footnotetext{
${ }^{3}$ For this specific example of next-to-leading form of the dispersion relation one might easily envision a quantumgravity theory which, even upon just a preliminary analysis of its general structure (for example considering unitarity issues), would safely prevent it. But this is precisely the point made in Ref. [36]: the possibility of large contributions from formally subleading terms introduces an extra layer of model dependence for the derivation of experimental bounds (for example quantum-gravity scenarios which, also inspired by Hawking's black-hole-based arguments, introduce some violation of unitarity are not uncommon). Quantum-gravity phenomenology has the difficult task of obtaining information relevant for research on the quantum-gravity problem in spite of the limited insight we presently have on the quantum-gravity problem. And the point articulated in this section concerns the widely-adopted procedure of truncating Planck-scale power series, as if it was possible to exclude that any term of higher order in the Planck length could not possibly matter. Some higher-order terms, which cannot be ruled out on the basis of our present understanding of the quantum-gravity problem, can instead give significant contributions.
} 
respect to the scenario that includes only the "leading-order term": for natural choices of values of parameters, such as $\zeta \sim \alpha \sim 1$, one finds [36] that, for the relevant Blazars, the implications of absorption of multi- $\mathrm{TeV}$ photons due to the pure-imaginary $E_{p}^{-3}$ turn out to be of the same order of magnitude of (but opposite to) the implications of the loss of pair-production absorption induced by the leading-order $E_{p}^{-1}$ term.

A similar argument can be applied to the analysis of the photopion production threshold relevant for the cosmic-ray spectrum, weakening the significance of bounds obtained used exclusively the structure of the spectrum near the GZK scale. However, in this cosmic-ray case there is an easy fix: instead of obtaining limits using exclusively the form of the spectrum near the GZK scale one should combine to it the analysis of the form of the spectrum in the neighborhood of "the ankle" which is the region where the threshold for another particle physics reaction leaves traces: the production of electron-positron pairs $p+\gamma \rightarrow e^{+}+e^{-}$. By studying thresholds at two different energy scales one can essentially remove concerns associated with the cubic term in Eq. (4.5) (if the coefficient is tuned to mask an effect at the GZK scale then it will not be significant at the ankle scale).

\section{Closing remarks}

Research reported over these past few year has shown that the phenomenology of Planckscale modifications of spacetime symmetries definitely has "the power of discovery": in several instances one finds that the data have sufficient sensitivity to confirm a relevant Planck-scale effect. And while most of the attention has somehow been directed toward effects that are only linearly penalized by the smallness of the Planck length, definitely there are cases in which good sensitivity to effects penalized quadratically by the Planck length is within reach [22, 24]. Positive (discovery) results of the relevant experimental searches would probably admit a rather straightforward interpretation.

And this phenomenology has shown the ability to falsify, although restricted to only a few examples. Particularly noteworthy from this perspective is the doubly-special-relativity idea, which could be fully falsified by a single measurement result, as discussed here in Subsection 3.2. It is of course disappointing that this phenomenology is still unable to falsify the theories that are the main focus of quantum-gravity research, such as (critical) String Theory and Loop Quantum Gravity, but this is not to be viewed as a weakness of the phenomenology, but rather as an indication of the lack of maturity of the relevant theoretical frameworks. In particular, for String Theory it is at present still unclear whether the framework is in principle falsifiable (does it make any definite predictions?), and of course the usual work of phenomenology, the one of finding practically doable experiments able to test the predictions of a given framework, is simply not possible, at least at present. The situation is (for very different reasons) equally disappointing on the side of attempting to falsify Loop Quantum Gravity: there it is probably reasonable to expect that the framework is indeed falsifiable, but we are presently (as a result of a much-discussed classical-limit problem) unable to obtain any definite predictions (we can still of course combine some heuristics with the few facts that are understood of Loop Quantum Gravity and gain some very valuable intuition for plausible quantum-gravity effects, but as long as these effects are not derived from the theory in 
a rigorously deductive manner they could not be used as basis for falsification of Loop Quantum Gravity).

Related (at least in part) to this impasse on the theory side are the difficulties (here stressed in Subsection 3.1 and Section 4) encountered in making good use of negative (no anomalous effects found) results obtained in this phenomenology. Ideally a phenomenologist would like its negative results (which of course end up being by far most numerous than positive ones) to be also very valuable, by placing strong intelligible constraints on models. Constraining certain types of models used in quantum-gravity research is a challenge for the same reasons I just discussed concerning falsification: for models/theories where "predictions" are not obtainable in a rigorously deductive manner (or simply there are no characteristic predictions at all) of course negative experimental results do not provide meaningful intelligible constraints. And, as illustrated by the concernes here briefly discussed in Section 4, even setting aside the "theory uncertainties" the phenomenology itself must face some challenges in making good use of negative results, mostly because data analyses capable to dig out such small effects are often vulnerable to contamination by other (possibly unwanted, from the perspective of the relevant analysis) small effects. Progress is likely to come at a rather slow pace, and only through the adoption and careful use of some well tailored test theories, in the sense here discussed in Subsection 3.1.

\section{References}

[1] G. Amelino-Camelia, gr-qc/0506117, Int. J. Mod. Phys. D14 (2005) 2167.

[2] N.R. Douglas and N.A. Nekrasov, Rev. Mod. Phys. 73, 977 (2001).

[3] S. Doplicher, K. Fredenhagen and J.E. Roberts, Phys. Lett. B331 (1994) 39.

[4] D.V. Ahluwalia, Phys. Lett. B339 (1994) 301.

[5] Y.J. Ng and H. Van Dam, Mod. Phys. Lett. A9 (1994) 335.

[6] G. Amelino-Camelia, gr-qc/9603014, Mod. Phys. Lett. A9 (1994) 3415; gr-qc/9603013; Mod. Phys. Lett. A11 (1996) 1411.

[7] L.J. Garay, gr-qc/9403008, Int. J. Mod. Phys. A10 (1995) 145.

[8] C. Rovelli, gr-qc/9710008, Living Rev. Rel. 1:1 (1998).

[9] L. Smolin, hep-th/0303185.

[10] S. Majid and H. Ruegg, Phys. Lett. B334 (1994) 348.

[11] J. Lukierski, H. Ruegg and W.J. Zakrzewski, Ann. Phys. 243 (1995) 90.

[12] G. Amelino-Camelia and S. Majid, hep-th/9907110, Int. J. Mod. Phys. A15, 4301 (2000).

[13] G.E. Volovik, gr-qc/0301043, Found. Phys. 33 (2003) 349.

[14] G. Chapline, E. Hohlfeld, R.B. Laughlin and D.I. Santiago, Phil. Mag. 81 (2001) 235; gr-qc/0012094; R.B. Laughlin, gr-qc/0302028, Int. J. Mod. Phys. A18 (2003) 831.

[15] G. Amelino-Camelia: gr-qc/0012051, Int. J. Mod. Phys. D11 (2002) 35; hep-th/0012238, Phys. Lett. B510 (2001) 255.

[16] G. Amelino-Camelia, "Are we at the dawn of quantum-gravity phenomenology?", gr-qc/9910089, Lect. Notes Phys. 541, 1 (2000). 
[17] S. Sarkar, gr-qc/0204092, Mod. Phys. Lett. A17 (2002) 1025.

[18] David Mattingly, Living Rev. Rel. 8:5 (2005).

[19] G. Amelino-Camelia, arXiv:0806.0339.

[20] G. Amelino-Camelia, J. Ellis, N.E. Mavromatos and D.V. Nanopoulos, hep-th/9605211, Int. J. Mod. Phys. A12 (1997) 607; G. Amelino-Camelia, J. Ellis, N.E. Mavromatos, D.V. Nanopoulos and S. Sarkar, astro-ph/9712103, Nature 393 (1998) 763.

[21] T. Kifune, Astrophys. J. Lett. 518 (1999) L21.

[22] R. Aloisio, P. Blasi, P.L. Ghia and A.F. Grillo, Phys. Rev. D62 (2000) 053010.

[23] R.J. Protheroe and H. Meyer: Phys. Lett. B493 (2000) 1.

[24] G. Amelino-Camelia and T. Piran, astro-ph/0008107, Phys. Rev. D64 (2001) 036005.

[25] R. Gambini and J. Pullin: Phys. Rev. D59 (1999) 124021 (1999).

[26] J. Alfaro, H.A. Morales-Tecotl and L.F. Urrutia, Phys. Rev. Lett. 84 (2000) 2318.

[27] G. Amelino-Camelia, gr-qc/0212002, New J. Phys. 6 (2004) 188.

[28] T. Jacobson, S. Liberati and D. Mattingly, hep-ph/0112207, Phys. Rev. D66 (2002) 081302.

[29] G. Amelino-Camelia: gr-qc/0107086, Phys. Lett. B528 (2002)181.

[30] T.J. Konopka and S.A. Major: New J. Phys. 4 (2002) 57.

[31] J.P. Norris, J.T. Bonnell, G.F. Marani and J.D. Scargle: astro-ph/9912136; A. de Angelis: astro-ph/0009271.

[32] U. Jacob and T. Piran, Nature Physics 3 (2007) 87.

[33] G. Amelino-Camelia, Nature Physics 3 (2007) 81.

[34] T. Jacobson, S. Liberati and D. Mattingly: arXiv.org/abs/astro-ph/0212190v2, Nature 424 (2003) 1019.

[35] L. Maccione, S. Liberati, A. Celotti and J.G. Kirk, arXiv:0707.2673, JCAP 0710 (2007) 013.

[36] G. Amelino-Camelia and L.F. Urrutia, in preparation. 